\title{
A Bargaining-Based Approach for Incentive-Compatible Message Forwarding in Opportunistic Networks
}

\author{
Fan $\mathrm{Wu}^{\dagger} \quad$ Tingting Chen ${ }^{\S}$ Sheng Zhong ${ }^{\star} \quad$ Chunming Qiao ${ }^{\star}$ Guihai Chen ${ }^{\dagger}$ \\ $\dagger$ Department of Computer Science and Engineering, Shanghai Key Laboratory of \\ Scalable Computing and Systems, Shanghai Jiao Tong University, China \\ $\S$ Department of Computer Science, Oklahoma State University, USA \\ ${ }^{\star}$ Department of Computer Science and Engineering, SUNY at Buffalo, USA \\ fwu@cs.sjtu.edu.cn; tingting@cs.okstate.edu; \{szhong, qiao\}@buffalo.edu; gchen@cs.sjtu.edu.cn
}

\begin{abstract}
Opportunistic networking is an important technique to enable users to communicate in an environment where contemporaneous end-to-end paths are unavailable or unstable. To support end-to-end messaging in opportunistic networks, a number of probabilistic routing protocols have been proposed. However, when nodes are selfish, they may not have incentives to participate in probabilistic routing, and the system performance will degrade significantly. In this paper, we present a novel incentive scheme for probabilistic routing that stimulates selfish nodes to participate. We not only rigorously prove the properties of our schemes, but also extensively evaluate our schemes using GloMoSim. Evaluation results show that there is an up to $\mathbf{7 5 . 8 \%}$ gain in delivery ratio compared with a probabilistic routing protocol providing no incentive.
\end{abstract}

\section{INTRODUCTION}

With the broad deployment of mobile wireless devices, opportunistic networking is becoming increasingly important in Mobile Ad-Hoc Networks (MANETs) and Delay-Tolerant Networks (DTNs), as well as mobile social networking applications. Opportunistic networking techniques enable users to communicate in an environment where contemporaneous end-to-end paths are unavailable or unstable. In such an environment, due to transitivity of links, messages are usually passed from one user to another in a store and forward fashion. Forwarding opportunities arise whenever mobile devices/users come into the communication range of each other. In contrast to traditional networking techniques, in which messages are delivered along preexisting end-to-end paths, opportunistic networking allows a message to be transferred from its source to its destination even when such a path from the source to the destination never exists.

In recent years, many routing protocols have been proposed to support end-to-end messaging in opportunistic networks (see [18] for a survey). A large portion of these existing protocols (e.g., [8], [27]) are probabilistic routing protocols. In probabilistic routing protocols, when a node carrying a message meets another node, it estimates the probability of the latter node being able to bring the message to the destination. This probability is used to decide whether the message should be forwarded to the latter node or not. Existing research has shown that probabilistic routing is a very practical and effective technique in opportunistic networking.

The adoption of such probabilistic routing protocols, however, might lead to reduced network performance when nodes have selfish behavior. In particular, opportunistic networks, like many distributed autonomous systems, suffer from common incentive problems such as the free-rider problem [2] when nodes are selfish. If selfish nodes are not appropriately rewarded, they do not have incentives to behave cooperatively. Hence, the performance of the network could degrade significantly, because only a small fraction of user nodes contribute their resources. Therefore, it is crucial to have a good incentive scheme that stimulates selfish nodes to cooperate in probabilistic routing.

Although much progress has been made in designing incentive schemes for wireless networks [10], most of existing incentive schemes are based on contemporaneous end-to-end connections, and thus do not apply to probabilistic routing. It is still an open problem to design incentive schemes for probabilistic routing. The objective of this paper is to study this open problem. Specifically, we would like to answer this question: if we are given a probabilistic routing protocol, how can we make it incentive compatible? That is, how can we enhance a probabilistic routing protocol such that selfish nodes will have incentives to cooperate when using this protocol?

To answer this question, we propose an approach based on bargaining. Our approach is motivated by the observation that message exchange in probabilistic routing is analogous to commodity exchange in markets: in probabilistic routing, a message is transferred from a node with a lower delivery probability of the message to a node with a higher delivery probability, just as in a market, a good is traded from a person with a lower valuation of the good to a person with a higher valuation. Therefore, if we can design a scheme, in which a node with a higher delivery probability can make profit by forwarding the packet from a node with a lower delivery probability, then the nodes will have incentives to participate in the packet forwarding. In this paper, we borrow ideas from bargaining theory [29], [30] to solve the incentive problem in 
probabilistic routing.

According to [1], to bargain is "to negotiate the terms of an agreement, as to sell or exchange". Bargaining theory captures both the competitive property and the positional property of a negotiation process between two or more parties. The intersecting yet conflicting interests of the different parties lead to a game between the involved parties, through which an agreement is reached.

In this paper, we model the message exchange process between a pair of nodes in probabilistic routing as a bargaining game. Hence, when a message is transferred from its source to its destination, it goes through a series of bargaining game. In each of these games, the message is traded from its current carrier to a node with even higher valuation. When this series of bargaining games is completed, the message reaches its destination. Based on analysis of the bargaining game, we design an incentive scheme that stimulates cooperative behavior in probabilistic routing. Below is a summary of our contributions in this paper:

- We model the process of probabilistic routing as a series of bargaining game, and show that, if no incentive scheme is used, participating in the game will not be to the nodes' best interests, and thus selfish nodes will refuse to participate, resulting that system performance will be degraded significantly.

- We present a novel and practical scheme to make a broad class of probabilistic routing protocols incentive compatible. We rigorously prove that, if our incentive scheme is used, there exists a unique subgame perfect equilibrium, in which nodes behave cooperatively. Here, intuitively, subgame perfect equilibrium means that a player can not get more benefit by unilaterally deviating from the equilibrium strategy in any subgame starting at her move. So, being cooperative is always to the best interests of the selfish nodes.

- We extend our model to consider the case in which there is a risk of breakdown in the bargaining process, and present a practical solution.

- We extensively evaluate our schemes using GloMoSim. Our evaluation results verify that, with our schemes, participation is to the best interest of each node, and there is an up to $75.8 \%$ gain in delivery ratio if compared with a probabilistic routing protocol having no incentive provided.

The rest of this paper is organized as follows. In Section II, we present technical preliminaries. In Section III, we show that, if no incentive scheme is used, participating in the game will not be to the nodes' best interests. Hence, we present our message trading scheme to achieve incentive compatibility for probabilistic routing. In Section IV, we extend our model to consider the risk of breakdown. In Section V, we discuss implementation issues. In Section VI, we report evaluation results on GloMoSim. In Section VII, we review related works. In Section VIII, we draw conclusions and discuss future work.

\section{TECHNICAL PRELIMINARIES}

Before presenting our system architecture and developing our scheme, we first review the probabilistic routing protocols we consider. We also review relevant game theoretic solution concepts.

\section{A. Basic Probabilistic Routing Protocol}

As we have mentioned, our target is to design an incentive scheme for a broad class of probabilistic routing protocols. Specifically, we assume we are given a probabilistic routing protocol, which is called the basic probabilistic routing protocol in the sequel. Then, starting from Section III, we establish our incentive scheme for this basic protocol to make it incentive compatible. Note that this basic probabilistic routing protocol can be one of many existing protocols, so that our scheme is widely applicable. Below we give a brief description of the type of basic probabilistic routing protocol we consider.

Probabilistic routing protocols are based on the observation that, in practice, nodes are not likely to move around randomly, but rather move in a predictable fashion based on mobility patterns. If a pair of nodes has met several times before, it is likely that they will meet again in the future. Such mobility patterns can be exploited to improve performance of routing protocol in opportunistic networks.

To exploit the mobility patterns, a probabilistic metric called delivery probability was introduced. Let $P_{a, b} \in[0,1]$ be the delivery probability from node $a$ to a destination node $b$. This metric indicates how likely that a node will be able to deliver a message to the destination. Each node stores a matrix of delivery probabilities. When two nodes meet, they exchange their delivery probability matrices. This matrix is used to update the internal delivery probability matrix. Then the delivery probability matrix is used to decide which message to forward from one node to another node.

Formally, a basic probabilistic routing protocol works as follows.

Forwarding Algorithm: When a node $a$ meets another node $b$, they perform a message exchange through a number of steps. First, node $a$ gives node $b$ a list of the messages node $a$ carries as well as their destinations. Each message is also annotated by $a$ with $a$ 's delivery probability. Node $a$ receives the same list from node $b$ and calculates its delivery probabilities of node $b$ 's messages. Node $a$ then requests from node $b$ the messages of which it has higher delivery probability than node $b$ by at least $\theta$.

Delivery Probability Calculation: The calculation of the delivery probability has two parts. The first part is to calculate each node's probability of meeting each of the other nodes. Let $\rho_{a, b}$ be the estimated probability that node $a$ and node $b$ meet. Here $\rho_{a, b}$ is computed based on the recorded movement events of the nodes during the last $\tau$ time slots, where a time slot is a fixed length of time (e.g., 1 hour or 1 day, depending on the movement speed of a typical node). The basic probabilistic 
routing protocol specifies a function $f_{1}()$, which computes

$$
\rho_{a, b}=f_{1}\left(\left\{e_{a, b}^{t-\hat{t}} \mid \hat{t} \in\{1,2, \ldots, \tau\}\right\}\right)
$$

where $t$ is the current time slot, $e_{a, b}^{t-\hat{t}}$ indicates whether node $a$ and node $b$ met in time slot $t-\hat{t}$.

Next, the transitive property of previously computed meeting probabilities is exploited to calculate the delivery probability $P_{a, b}$. The basic probabilistic routing protocol also specifies a function $f_{2}()$, which computes

$$
P_{a, b}=f_{2}\left(\left\{\left(a, b, \rho_{a, b}\right) \mid a, b \in V\right\}\right),
$$

where $V$ is the set of nodes in the system.

Note that, by using different functions for $f_{1}()$ and $f_{2}()$, we can get different instances of basic probabilistic routing protocol. For example, PROPHET [27] and MV [8] are both instances of the basic probabilistic routing protocol. Protocols in [12], [13] are also instances of this class after scaling the estimation to the range $[0,1]$.

As we have mentioned, the existing probabilistic routing protocols lack incentive mechanisms, and accordingly selfish intermediate nodes may not be willing to forward messages for others for free. In some applications, incentives are needed in order to stimulate the selfish nodes to forward messages, so that messages are forwarded to the node with a higher and higher delivery probability and finally to the destination.

\section{B. Game Theoretic Model and Solution Concepts}

To study the incentive compatibility of probabilistic routing, we model the message forwarding process as a bargaining game [30].

Specifically, we isolate a pair of nodes, who come into the communication range of each other, and model the interaction between them for the possible transfer of a message as a bargaining game. One of these two nodes is the current carrier of the message. It determines whether to forward the message to the other node. We view this process as bargaining, where the current carrier of the message is the seller of the message, and the other node is the buyer. Hence, there are two players in the game, the seller $S$ and the buyer $B$. The set of players is $N=\{S, B\}$. These two players need to agree on a price at which $S$ sells the message to $B$.

The bargaining game is played in rounds. In each round, the seller $S$ makes a proposal, then the buyer $B$ decides to accept it or not. Acceptance ends the game while rejection leads to the next round. A strategy $s_{i}$ of player $i \in N$ is a function that assigns an action to player $i$ when it is its turn to move. As a notational convention, $-i$ represents the player other than player $i$ in the bargaining game. Similarly, $s_{-i}$ represents the strategies of the player other than player $i$. Note that $s=\left(s_{i}, s_{-i}\right)$ is a strategy profile.

If an agreement on purchase price $x$ is reached in round $r$, then the two players' utilities are:

$$
\begin{aligned}
& u_{S}=x-V_{S}(m)-T(m)-c_{S}(r), \\
& u_{B}=V_{B}(m)-P(m)-x-c_{B}(r),
\end{aligned}
$$

where $V_{i}(m)$ is the valuation of message $m$ to player $i, T(m)$ and $P(m)$ are the costs associated with the transmission and reception of message $m, c_{S}(r)$ and $c_{B}(r)$ are the bargaining costs of seller $S$ and buyer $B$ in the procedure of the game.

We note that $V_{i}(m)$ is the integrated message valuation of node $i$ and its downstream nodes. In a bargaining game, if $-i$ is $i$ 's downstream node, then $V_{-i}(m)-V_{i}(m)-T(m)-P(m)$ is this game's profit margin. ${ }^{1} V_{i}(m)$ is determined as follows: assume that whoever delivers message $m$ to the destination node can get a payment $\omega$ from the source node. Considering the previously defined delivery probability $P_{i, d}$, the valuation of a message $m$ at node $i$ is as follows.

$$
V_{i}(m)=\omega \cdot P_{i, d} .
$$

Clearly, a node with a higher delivery probability of a message also has a higher valuation of the message. Hence, a node has incentives to purchase the message from nodes who have lower delivery probabilities. (Consequently, the message is forwarded to the node with higher and higher delivery probability, and finally reaches the destination.) For simplicity, we assume that the source and the destination nodes in a session are trustworthy, and do not consider their utilities in this work. However, we will consider the case in which both the source and the destination nodes act as game players in our future work.

Assume that each of the two player nodes $S$ and $B$ incurs a cost $\sigma>0$ for every round of the game. Then we have $c_{S}(r)=c_{B}(r)=r \cdot \sigma$.

To enable nodes to pay each other, just as in [4], [14], [40], [41], [43]-[45], we assume that there is some kind of virtual currency in the system. In the system, there is a Credit Clearance Center (CCC). Each node has an account in the $\mathrm{CCC}$ and each transaction has to be processed by the CCC. Each node keeps a digitally signed recept for each transaction and submit receipts to the CCC. The CCC is a server connected to the Internet. So the node can access the $\mathrm{CCC}$ whenever they have connections to the Internet. The CCC clear the transaction after receiving the receipts.

Let $R$ be the maximum number of rounds for bargaining. If the players do not reach any agreement after $R$ rounds of bargaining, then their utilities are

$$
\begin{aligned}
& u_{S}=-c_{S}(R), \\
& u_{B}=-c_{B}(R) .
\end{aligned}
$$

In Section III, we assume that the value of $R$ is known by all nodes. ${ }^{2}$ We also assume that the players always keep bargaining for the possible message exchange, until an agreement is reached or the bargaining reaches the last round. In Section IV, we study the case in which nodes do not know the value of $R$, and can terminate the bargaining game to avoid loss exaggeration.

\footnotetext{
${ }^{1}$ We do not deduct the bargaining costs here, because they are variables depending on the design of the bargaining scheme.

${ }^{2}$ This is the case when, for example, nodes are equipped with GPS systems, which enable they to calculate the length of communication time by geometry, given communication ranges, speeds, and heading directions of the two nodes.
} 
Bargaining game is a special case of extensive game with perfect information [30]. In an extensive game with perfect information $\Gamma$, a history $h$ is a sequence of actions starting from the beginning of the game. A subgame is the remaining part of the game following a specific history. Denote by $\left.\Gamma\right|_{h}$ the subgame that follows the history $h$. Let $\left.s_{i}\right|_{h}$ denote the strategy that $s_{i}$ induces in the subgame $\left.\Gamma\right|_{h}$, and $\left.u_{i}\right|_{h}$ denote the utility of player $i$ in subgame $\left.\Gamma\right|_{h}$. We now review commonly used solution concepts for extensive game with perfect information.

In extensive games, an important solution concept is subgame perfect equilibrium [30]:

Definition 1 (Subgame Perfect Equilibrium): A subgame perfect equilibrium of an extensive game with perfect information $\Gamma$ is a strategy profile $s^{\star}$ such that for every player $i \in N$ and every nonterminal history $h$, after which it is player $i$ 's turn to take an action, we have

$$
\left.u_{i}\right|_{h}\left(\left.s_{i}^{\star}\right|_{h},\left.s_{-i}^{\star}\right|_{h}\right) \geq\left. u_{i}\right|_{h}\left(s_{i},\left.s_{-i}^{\star}\right|_{h}\right),
$$

for every strategy $s_{i}$ of player $i$ in the subgame $\left.\Gamma\right|_{h}$.

The game studied in this paper has a finite horizon, which means that the number of rounds is finite and the number of actions at any round is finite. An efficient tool to verify a strategy profile $s^{\star}$ is a subgame perfect equilibrium in a game with a finite horizon is the one deviation property [30]:

Lemma 2 (The One Deviation Property): The strategy profile $s^{\star}$ is a subgame perfect equilibrium of a finite horizon extensive game with perfect information $\Gamma$ if and only if for every player $i \in N$ and every history $h$, after which it is player $i$ 's turn to take an action, we have

$$
\left.u_{i}\right|_{h}\left(\left.s_{i}^{\star}\right|_{h},\left.s_{-i}^{\star}\right|_{h}\right) \geq\left. u_{i}\right|_{h}\left(s_{i},\left.s_{-i}^{\star}\right|_{h}\right),
$$

for every strategy $s_{i}$ of player $i$ in the subgame $\left.\Gamma\right|_{h}$ that differs from $\left.s_{i}^{\star}\right|_{h}$ only in the action it prescribes after the initial history of $\left.\Gamma\right|_{h}$.

We can restrict our attention, for each player $i$ and each subgame, to alternative strategies that differ from $s_{i}^{\star}$ in the actions they prescribe after just one history. That is to say, a strategy profile is a subgame perfect equilibrium if and only if in each subgame the player who makes the first move cannot obtain a better utility by changing only his initial action.

\section{Incentive Analysis And Design of Scheme}

In this section, we first show that, if there is no incentive scheme, nodes will be unwilling to participate. Then we propose a message trading scheme, under which the system will converge to a unique subgame perfect equilibrium. In this subgame perfect equilibrium, an agreement between the two players is reached immediately in the first round, so that the message can be forwarded from the seller to the buyer without any hassle.

\section{A. Analysis of the System without Scheme}

In the section, we use a standard analysis method from bargaining theory to show that the buyer cannot get any benefit from the trading, if no incentive scheme is provided.
Recall that, in each round $r$, the seller $S$ makes a proposal $x$ and the buyer decides whether to accept it or not; and the bargaining process lasts until an agreement is reached or the bargaining reaches the last round $R$. Then, if we analyze the bargaining process backwards, starting from the last round, we can conclude that the best strategy for the seller $S$ is to always propose

$$
x(r)=V_{B}(m)-P(m)+(R-r) \cdot \sigma,
$$

in round $r$. Suppose the buyer $B$ prefers reaching an agreement to disagreement when the above two possibilities have the same utility to her. Since the buyer $B$ wants to maximize her utility, she will accept the offer

$$
x \leq V_{B}(m)-P(m)+(R-r) \cdot \sigma,
$$

in round $r$ and reject the others. According to the one deviation property, this strategy profile is a subgame perfect equilibrium. In this subgame perfect equilibrium, an agreement is reached in the first round. The seller $S$ gets good utility:

$$
\begin{aligned}
u_{S} & =x-V_{S}(m)-T(m)-\sigma \\
& =V_{B}(m)-V_{S}(m)-T(m)-P(m)+(R-2) \cdot \sigma .
\end{aligned}
$$

(We assume $V_{B}(m)>V_{S}(m)+T(m)+P(m)$ and $R>2$.) But the buyer $B$ 's utility is

$$
\begin{aligned}
u_{B} & =V_{B}(m)-P(m)-x-\sigma \\
& =-R \cdot \sigma .
\end{aligned}
$$

The above analysis shows that, if there is no incentive scheme, the buyer $B$ always gets negative utility no matter an agreement is reached or not. This result inevitably hurts the buyer's incentive to buy the message. In reality, selfish nodes will refuse to participate in the game to avoid losses. So a scheme for message trading, which stimulates nodes to participate in the game, is highly needed.

\section{B. Message Trading Scheme}

In this section, we propose a scheme that stimulates nodes to participate in the game, so that messages can be forwarded.

The main idea of our scheme is to influence the players' strategy by introducing a carefully designed transaction fee. Denote by $X(m, x)$ the transaction fee of the message $m$ at price $x$. In the message trading game, this transaction fee is included in the final purchase price. That is to say, the seller gives out some of her profit as transaction fee when accessing the CCC to clear the transaction. The advantage of introducing the transaction fee is that, by carefully choosing a formula for $X(m, x)$, we can change the seller's best strategy in the game, such that her offer in the first round is a "reasonable" price for the message. This price is "reasonable" in the sense that it makes the transaction profitable for both parties. That is, both parties will have positive utilities in the game. ${ }^{3}$ Furthermore,

\footnotetext{
${ }^{3}$ Recall that a node request a message on which it has at least $\theta$ higher delivery probability. In our scheme, a message trading game takes place only on a message $m$ such that $V_{B}(m)-V_{S}(m)-T(m)-P(m)>2 \sigma$.
} 
accepting this "reasonable" price is also to the best interest of the buyer.

Our designed formula for $X(m, x)$ is as follows:

$X(m, x)=\left\{\begin{array}{l}\gamma \quad \text { if } x \leq \frac{V_{S}(m)+V_{B}(m)+T(m)-P(m)}{2} \\ k\left(x-\frac{V_{S}(m)+V_{B}(m)+T(m)-P(m)}{2}\right)+\gamma \quad \text { o.w. }\end{array}\right.$

where $\gamma \leq\left(V_{B}(m)-V_{S}(m)-T(m)-P(m)\right) / 2-\sigma$ is a very small primary transaction fee, and $k=2-2 \gamma /\left(V_{B}(m)-\right.$ $\left.V_{S}(m)-T(m)-P(m)\right)$. We note that the advantage of using the proposed formula is twofold. On one hand, since $\gamma$ is very small, the transaction fee is also very small compared with the utilities got by the players, when the purchase price is in reasonable range $\left(x \leq\left(V_{S}(m)+V_{B}(m)+T(m)-P(m)\right) / 2\right)$. On the other hand, using the proposed formula, the equilibrium purchase price is $\left(V_{S}(m)+V_{B}(m)+T(m)-P(m)\right) / 2$, which makes the utility difference between seller and buyer is only $\gamma$. When $\gamma$ converges to 0 , seller and buyer will have the same utility in the transaction.

Our analysis in Section III-C will explain why it is a good formula for the transaction fee in detail. Figure 1 gives a complete description of our scheme.

Suppose two nodes come into the communication range of each other.

1) The two nodes exchange the lists of the message they carry. Suppose one of the nodes (buyer $B$ ) wants to buy a message $m$ from the other node (seller $S$ ).

2) In each round $r \leq R$, starting from $r=1$, the seller $S$ makes a proposal (a purchase price) $x$, which the buyer $B$ then either accepts or rejects. Acceptance ends the game while rejection leads to round $r+1$.

3) If an agreement is reached, the seller $S$ transmits the message $m$ to the buyer $B$; and the buyer $B$ pays $x$ to the seller $S$. If no proposal is ever accepted then the outcome is the disagreement event.

4) When the seller $S$ has a connection to the credit clearance center (CCC), it clear the transaction and pays transaction fee $X(m, x)$.

Fig. 1. Message Trading Scheme.

\section{Analysis of Our Scheme}

For notational clearance, we let $u_{i}^{r}$ denote player $i$ 's utility if an agreement is reached in round $r$.

Suppose an agreement is reached in round $r$. The utilities of the seller $S$ and the buyer $B$ become:

$$
\begin{aligned}
& u_{S}^{r}=x-X(m, x)-V_{S}(m)-T(m)-r \cdot \sigma, \\
& u_{B}^{r}=V_{B}(m)-P(m)-x-\sigma \cdot r .
\end{aligned}
$$

If the disagreement event is reached, then the utilities of the players are

$$
u_{S}^{D}=u_{B}^{D}=-R \cdot \sigma .
$$

We assume that each player $i \in\{S, B\}$ prefers reaching an agreement in round $r$ to round $r+1$, when $u_{i}^{r}=u_{i}^{r+1}$; and prefers reaching an agreement in the last round to disagreement event, when $u_{i}^{R}=u_{i}^{D}$. Then, we have the following theorem.

Theorem 3: If the above scheme is used, then there exists a unique subgame perfect equilibrium. In the subgame perfect equilibrium, the seller $S$ always proposes

$$
x^{\star}=\frac{V_{S}(m)+V_{B}(m)+T(m)-P(m)}{2},
$$

in each round $r$; the buyer $B$ only accepts proposal $x$ for which

$$
x \leq \begin{cases}\frac{V_{S}(m)+V_{B}(m)+T(m)-P(m)}{2}+\sigma & \text { if } r<R \\ V_{B}(m)-P(m) & \text { if } r=R,\end{cases}
$$

and rejects any other proposals.

Proof: We construct a subgame perfect equilibrium by backwards induction.

Start from the last round $r=R$. The last mover is the buyer $B$. She will accept proposal $x$ only if the outcome is not worse than the disagreement event.

$$
\begin{aligned}
u_{B}^{R} & \geq u_{B}^{D} \\
V_{B}(m)-P(m)-x-R \cdot \sigma & \geq-R \cdot \sigma \\
x & \leq V_{B}(m)-P(m) .
\end{aligned}
$$

Next, we consider the move of seller $S$. Since $S$ is selfish, it must make a proposal $x^{\star}(R)$ that maximize its utility in round $R$.

$$
\begin{aligned}
& x^{\star}(R) \\
& =\underset{x}{\operatorname{argmax}}\left(u_{S}^{R}\right) \\
& =\underset{x}{\operatorname{argmax}}\left\{\begin{array}{rr}
x-X(m, x)- & V_{S}(m)-T(m)-R \cdot \sigma \\
-R \cdot \sigma & \text { if } x \leq V_{B}(m)-P(m) \\
- & \text { if } x>V_{B}(m)-P(m) .
\end{array}\right.
\end{aligned}
$$

Here $V_{S}(m), T(m), P(m)$, and $R \cdot \sigma$ are constants. We consider

$$
\begin{aligned}
& x-X(m, x) \\
= & \left\{\begin{array}{l}
x-\gamma \quad \text { if } x \leq \frac{V_{S}(m)+V_{B}(m)+T(m)-P(m)}{2} \\
x-k\left(x-\frac{V_{S}(m)+V_{B}(m)+T(m)-P(m)}{2}\right)-\gamma \quad \text { o.w., }
\end{array}\right.
\end{aligned}
$$

Since $k>1, x-X(m, x)$ get maximized when $x=\left(V_{S}(m)+\right.$ $\left.V_{B}(m)+T(m)-P(m)\right) / 2$. Consequently,

$$
x^{\star}(R)=\frac{V_{S}(m)+V_{B}(m)+T(m)-P(m)}{2} .
$$

Next, move to the round $r=R-1$. The buyer $B$ accepts a proposal $x$ only when

$$
\begin{gathered}
u_{B}^{R-1} \geq u_{B}^{R} \\
V_{B}(m)-P(m)-x-(R-1) \cdot \sigma \\
\geq V_{B}(m)-P(m)-x^{\star}(R)-R \cdot \sigma
\end{gathered}
$$




$$
x \leq \frac{V_{S}(m)+V_{B}(m)+T(m)-P(m)}{2}+\sigma=\beta .
$$

Again, the seller $S$ makes a proposal $x^{\star}(R-1)$ that maximize its utility.

$$
\begin{aligned}
& x^{\star}(R-1) \\
= & \underset{x}{\operatorname{argmax}}\left(u_{S}^{R-1}\right) \\
= & \underset{x}{\operatorname{argmax}}\left\{\begin{array}{cc}
x-X(m, x)-V_{S}(m)-T(m) & \\
-(R-1) \sigma & \text { if } x \leq \beta \\
u_{S}^{R} & \text { if } x>\beta
\end{array}\right. \\
= & \frac{V_{S}(m)+V_{B}(m)+T(m)-P(m)}{2} .
\end{aligned}
$$

Repeat the above analysis backwards to the first round. In each round $r<R$, the buyer $B$ 's optimal strategy is accepting proposal $x$ for which

$$
x \leq \frac{V_{S}(m)+V_{B}(m)+T(m)-P(m)}{2}+\sigma,
$$

and the seller $S$ 's optimal strategy is proposing

$$
x^{\star}(r)=\frac{V_{S}(m)+V_{B}(m)+T(m)-P(m)}{2} .
$$

Since in every subgame, the constructed strategy is strictly optimal, the game has a unique subgame perfect equilibrium. This completes our proof.

Note that the structure of message trading game allows the game to continue for $R$ rounds, but under our scheme, an agreement is reached immediately at price $x=\left(V_{S}(m)+\right.$ $\left.V_{B}(m)+T(m)-P(m)\right) / 2$ in the subgame perfect equilibrium. (One may wonder why we need the sophisticated analysis of the $R$-round game while under our scheme the agreement is reached in just one round. The answer is that reaching an agreement in one round is just the equilibrium state of the Rround game. Without analysis of the R-round game, we would not be able to determine it is an equilibrium. This explanation also applies to a similar question for our work in Section IV.)

\section{RISK OF BREAKDOWN}

In the previous section, we have considered the case in which the player nodes know $R$, which is the maximum number of rounds. In this section, we extend our work to consider the case in which player nodes do not know the exact number of rounds in the game. This happens when nodes do not know when they will go out of the communication range of each other. Consequently, there is a risk of breakdown during both the bargaining phase and the message transmission phase.

\section{A. Extended Model}

To study the above scenario, we need an extended model for our game. Here we use the model of bargaining with a risk of breakdown [29], [30]. Specifically, we assume that immediately after the buyer rejects any offer in any round, with probability $p(0<p<1)$ the bargaining breaks down in disagreement, and with probability $1-p$ the game proceeds to the next round. We also assume that the probability of breakdown during message transmission increases with the advance of bargaining. That is to say, the longer the bargaining phase takes, the higher risk of breakdown the message transmissions have. Let $q(r)$ be the probability of breakdown during message transmissions if the agreement could be reached in round $r$. Here $q(r)$ is an increasing function on range $[0,1]$.

Different from the bargaining game model we have used in Section III, there is no explicit end of the game in the extended game model with risk of breakdown. The bargaining game may last so long that the gain from trading the message cannot cover the bargaining cost and the transmission cost. To avoid this situation, we allow the players to terminate the game when they realize that the loss will be widened if bargaining continues.

If an agreement on purchase price $x$ is reached in round $r$, then the two players' expected utilities are:

$$
\begin{aligned}
u_{S}^{r} & =(1-q(r))\left(x-X(m, x)-V_{S}(m)\right)-T(m)-r \cdot \sigma, \\
u_{B}^{r} & =(1-q(r))\left(V_{B}(m)-x\right)-P(m)-r \cdot \sigma .
\end{aligned}
$$

If the bargaining terminates in round $r$ or breaks down after round $r$, then the players' utilities are:

$$
u_{S}^{D}=u_{B}^{D}=-r \cdot \sigma
$$

\section{B. Bargaining Scheme for Extended Model}

Suppose the bargaining scheme in Figure 1 is still used in the extended model. We note that previously defined transaction fee no longer works well, because the seller's best expected utility

$$
u_{S}^{r}<0
$$

when

$$
q(r)>1-\frac{2(T(m)+r \cdot \sigma)}{V_{B}(m)-V_{S}(m)+T(m)-P(m)-2 \gamma} .
$$

This prevents the players from reaching an agreement, although there still exist prices at which both of the players can get profit. Consequently, previously proposed scheme need to be changed to work with the extended model.

To cope with the extended model, we continue to use the scheme in Figure 1 with a changed transaction fee formula as follows:

$X^{\prime}(m, x)=\left\{\begin{array}{l}\gamma \quad \text { if } x \leq \frac{V_{S}(m)+V_{B}(m)+\frac{T(m)-P(m)}{1-q(r)}}{2} \\ k\left(x-\frac{\left.V_{S}(m)+V_{B}(m)+\frac{T(m)-P(m)}{1-q(r)}\right)+\gamma}{2} \text { o.w., }\right.\end{array}\right.$

where

$$
\gamma \leq\left(V_{B}(m)-V_{S}(m)-\frac{T(m)-P(m)}{1-q(r)}\right) / 2-\sigma
$$

is the primary transaction fee, and

$$
k=2-2 \gamma /\left(V_{B}(m)-V_{S}(m)-\frac{T(m)-P(m)}{1-q(r)}\right) .
$$

Recall that the players can terminate the bargaining when their losses will definitely be widened, if bargaining continues. 
The seller terminates the bargaining when the expected gain from the trade cannot cover the transmission cost:

$$
\begin{aligned}
& (1-q(r))\left(x-X^{\prime}(m, x)-V_{S}(m)\right)<T(m) \\
\Rightarrow & \frac{V_{S}(m)+V_{B}(m)+\frac{T(m)-P(m)}{1-q(r)}}{2}-\gamma-V_{S}(m)<\frac{T(m)}{1-q(r)} \\
\Rightarrow & r>q^{-1}\left(1-\frac{T(m)+P(m)}{V_{B}(m)-V_{S}(m)-2 \gamma}\right) .
\end{aligned}
$$

While the buyer terminates the bargaining when she cannot get satisfying proposal and her maximal gain in the next round cannot cover expected further bargaining cost:

$$
\begin{aligned}
& (1-q(r+1))\left(V_{B}(m)-x\right)-P(m)<(1-p) \sigma \\
\Rightarrow & \frac{V_{B}(m)-V_{S}(m)-\frac{T(m)-P(m)}{1-q(r+1)}}{2}<\frac{P(m)+(1-p) \sigma}{1-q(r+1)} \\
\Rightarrow & r>q^{-1}\left(1-\frac{T(m)+P(m)+2(1-p) \sigma}{V_{B}(m)-V_{S}(m)-\gamma}\right)-1 .
\end{aligned}
$$

Let

$$
\begin{aligned}
& R^{1}=\left\lceil q^{-1}\left(1-\frac{T(m)}{V_{B}(m)-V_{S}(m)-2 \gamma}\right)\right\rceil, \\
& R^{2}=\left\lceil q^{-1}\left(1-\frac{T(m)+P(m)+2(1-p) \sigma}{V_{B}(m)-V_{S}(m)-\gamma}\right)-1\right] .
\end{aligned}
$$

Consequently, the last round $R=\min \left(R^{1}, R^{2}\right)$.

Theorem 4: There exists a unique subgame perfect equilibrium in extended model. In the subgame perfect equilibrium, the seller $S$ always proposes

$$
x^{\triangle}=\frac{V_{S}(m)+V_{B}(m)+\frac{T(m)-P(m)}{1-q(r)}}{2},
$$

in each round $r<R^{1}$; and terminates the bargaining if the game forward to round $r=R$ (if $R=R^{1}$ ). The buyer $B$ only accepts proposal $x$ for which

$$
x \leq\left\{\begin{array}{cc}
V_{B}(m)-\frac{(1-p)(1-q(r+1))\left(V_{B}(m)-V_{S}(m)\right)}{2(1-q(r))} & \\
+\frac{(1-p)(T(m)+P(m)+2 \sigma)}{2(1-q(r))} & \text { if } r<R \\
V_{B}(m)-P(m) & \text { if } r=R ;
\end{array}\right.
$$

and terminates the bargaining if she cannot accept the proposal in round $r=R$ (if $R=R^{2}$ ).

The proof of Theorem 4 is similar to that of Theorem 3 . Due to limitation of space, we omit the proof here.

Note that under our scheme, an agreement is reached immediately at price $x=\left(V_{S}(m)+V_{B}(m)+\frac{T(m)-P(m)}{1-q(r)}\right) / 2$ in the subgame perfect equilibrium.

\section{IMPLEMENTATION ISSUES}

Given the preceding core theoretical results, we now discuss some implementation issues related to integrating the proposed incentive scheme into a given probabilistic routing protocol. Due to space limitations, we only focus on a high-level overview.
Misreporting Prevention: The incentive-compatibility provided by our message trading scheme relies on the truthfulness of the underlying probabilistic routing protocol. However, a selfish node may lie about her meeting probabilities with and delivery probabilities to the other nodes, in order to pay less or charge more for messages. Therefore, we must ensure that each node honestly reports her meeting probabilities and delivery probabilities.

We prevent the misreporting by requiring each forwarding node to submit the delivery probabilities of herself and her downstream node together with the transaction receipt of each message to the CCC. In this way, for each message, the CCC will collect two delivery probabilities for each forwarder: one is from the forwarder herself, the other is from her upstream node. On one hand, reporting a lower delivery probability for a message is not beneficial to a forwarder herself. On the other hand, a forwarder's delivery probability for a message cannot be increased unless she meets a node with a higher delivery probability, in which case the message should be forwarded to the later node. Consequently, the forwarder should not report a higher delivery probability for the message. Therefore, a forwarder's delivery probability for a message reported by herself should be no more than that reported by her upstream node. If aging is used in calculating the delivery probability, after aging, the delivery probability with a later time stamp should be no more than that with an earlier time stamp. Once a misreporting is detected, indicating that one of the two nodes (the forwarder and her upstream node) must have manipulated the transaction, the CCC will punish both of the two nodes with a high penalty. Intuitively, this method can prevent the nodes from misreporting, because a node cannot increase her utility, by unilaterally misreporting her delivery probability for a message, given the delivery probabilities for the message of her upstream node and downstream node.

Message Duplication Prevention: A node may forward a message to multiple downstream nodes, in order to get more utility. Such a misbehavior can inject redundant messages into the network, thus the performance of the network may be degraded due to an increased workload. We solve this problem by letting the CCC only process one transaction recept from each node for each message. To prevent a node selectively submit the most beneficial recept from a number of transactions of the same message, the CCC also check the receipts from the other nodes. A duplicated message must have multiple receipts indicating the same upstream node from different nodes. If a message duplication behavior is detected, the CCC will punish the misbehaving node with a high penalty.

Forwarder Selection: When our message trading schemes are used, to maximize utility, a rational strategy of a node is to always forward the message to the node with the highest delivery probability to her knowledge. This rational strategy does not contradict to the objective of our system, and can usually increase system performance. In this work, we assume that the nodes do not have the knowledge on delivery probabilities of nodes possibly met in the future, and do not 


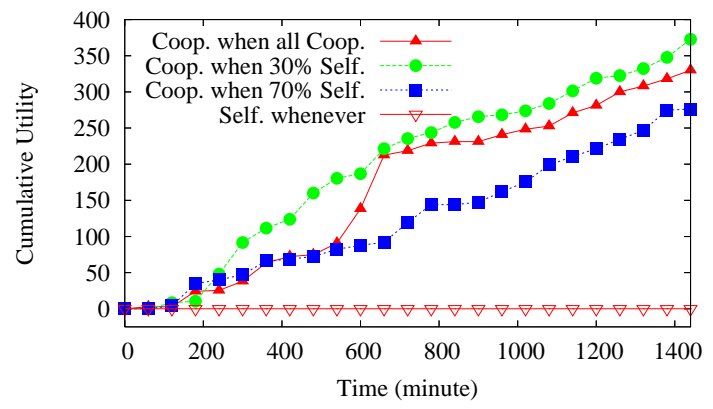

(a) No breakdown.

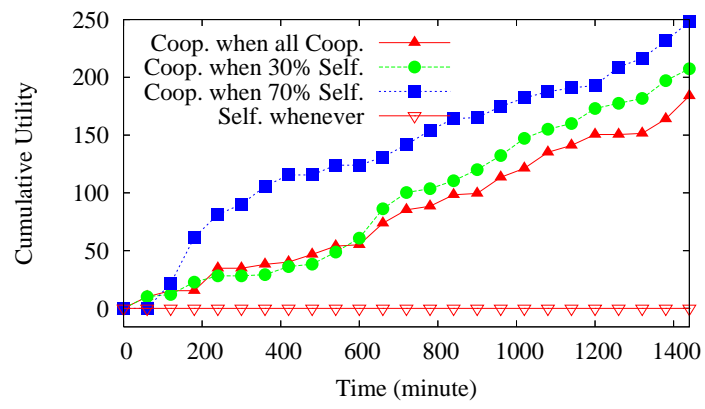

(b) Having risk of breakdown.

Fig. 2. Cumulative utilities obtained by node 13 in a network with 20 nodes. Four cases are compared: A) Behaving cooperatively when all the other nodes are cooperative; B) Behaving cooperatively when $30 \%$ nodes are selfish; C) Behaving cooperatively when $70 \%$ nodes are selfish; D) Behaving selfishly no matter what the others do. The figures demonstrate that the node get better utility when behaving cooperatively.

have patience to wait for another node with a potentially higher delivery probability. However, we will study how to provide the incentives for opportunistic routing protocols, when the nodes are capable of deciding whether to start a message bargaining game with the current buyer in our future work.

\section{EVALUATIONS}

In this section, we integrate our schemes with MV routing [8] and evaluate them using GloMoSim [38]. Our evaluations have two objectives. One is to verify that our schemes indeed prevent nodes from being selfish. The other is to measure the influence of our schemes on the delivery ratio of probabilistic routing in a wireless network with selfish nodes.

\section{A. Methodology}

We consider wireless networks with 10, 20, 30, and 40 mobile nodes randomly distributed in a terrain area of 10 $\mathrm{km}$ by $10 \mathrm{~km}$. Each node has three locations in the physical terrain, and randomly travel among these locations at a speed uniformly chosen between $10 \mathrm{~m} / \mathrm{s}$ and $30 \mathrm{~m} / \mathrm{s}$. After reaching its destination, the node stays there for 5 minutes. ${ }^{4}$ Nodes use IEEE 802.11 (at $11 \mathrm{Mbps}$ ) as the MAC layer protocol. The radios' transmission range is set to 250 meters. Nodes broadcast hello message every 1 second. The length of time unit used in probabilistic routing protocols is set to 1 minute.

Nodes generate messages with uniform time interval of 10 minutes. The destination of the message is randomly selected from the other nodes. A message is dropped if it can not be forwarded to another node in 1 hour. Each simulation runs for 24 hours, and is repeated 10 times with different random seeds. Every node has an initial credit of 5000, and pays 100 credit for each delivered message.

In the model with risk of breakdown, we set $p=0.01$ and $q(r)=0.1+0.01(r-1)$, where $r \geq 1 .^{5}$

\footnotetext{
${ }^{4} \mathrm{We}$ evaluate the performance of our schemes on a 3-waypoint mobility model instead of human movement traces, because the time spans of available human movement traces are not long enough for this evaluation. The range of movement speed roughly captures the average driving speeds in city.

${ }^{5}$ Here $q(r)$ is not limited to linear function. It can also be quadratic function, reciprocal function, exponential function, etc. However, the evaluation results of using different functions for $q(r)$ are identical. Therefore, we only show the results for linear $q(r)$ in this paper.
}

Node Behaviors: In our evaluations, we compare two types of node behavior:

- Cooperative behavior: Following the scheme faithfully.

- Selfish behavior: As we have mentioned in previous sections, selfish nodes are not be willing to participate in message forwarding for others. We report results when $30 \%$ and $70 \%$ of the nodes are selfish.

Metrics: We evaluate three metrics:

- Cumulative utility: The total utility obtained by a node over a period of time. Cumulative utility reflects the impacts of a node's behavior on its own. All nodes prefer a higher utility.

- Credit balance: The credit remained on a node. Credit balance reflects a node's participation in the process of message forwarding. All nodes prefer a higher credit balance.

- Delivery ratio: The ratio between number of delivered messages and total generated messages. Delivery ratio reflects the impacts of our schemes on the performance of a opportunistic network with selfish nodes.

\section{B. Impacts of Selfish Behavior on Cumulative Utility and Credit Balance}

In our first set of evaluations we demonstrate that being cooperative is better than being selfish in terms of cumulative utility and credit balance.

Figure 2 shows the cumulative utilities of node 13 in a 20-node network, with and without a risk of breakdown. We note that the results for the other players are similar to that of player 13. In the figures, four different cases are compared: A) Behaving cooperatively when all the other nodes are cooperative; B) Behaving cooperatively when $30 \%$ nodes are selfish; C) Behaving cooperatively when $70 \%$ nodes are selfish; D) Behaving selfishly no matter what the other nodes do. We can see that the node always has positive and increasing cumulative utility when it behaves cooperatively no matter what the other nodes do. In contrast, the node's cumulative utility always stays at 0 throughout the simulation if it behaves selfishly. Therefore, for an individual node, being cooperative can always get a better utility than being selfish. 


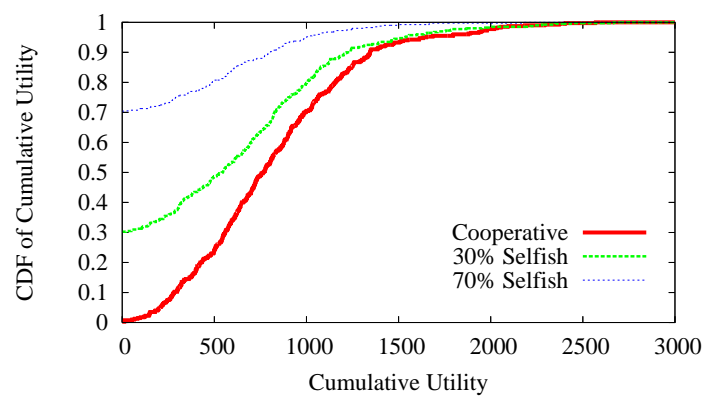

(a) No breakdown.

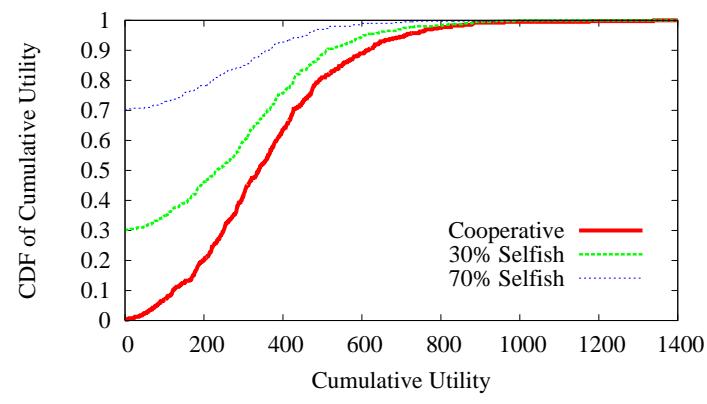

(b) Having risk of breakdown.

Fig. 3. CDF of cumulative utilities achieved with our schemes for 400 tracked node records. (The simulation on a network with 40 nodes is repeated 10 times with different random seeds.) Three cases are compared: A) $100 \%$ nodes behave cooperatively; B) $30 \%$ nodes behave selfishly; C) $70 \%$ nodes behave selfishly. The figures show that generally nodes get higher utilities when being cooperative.

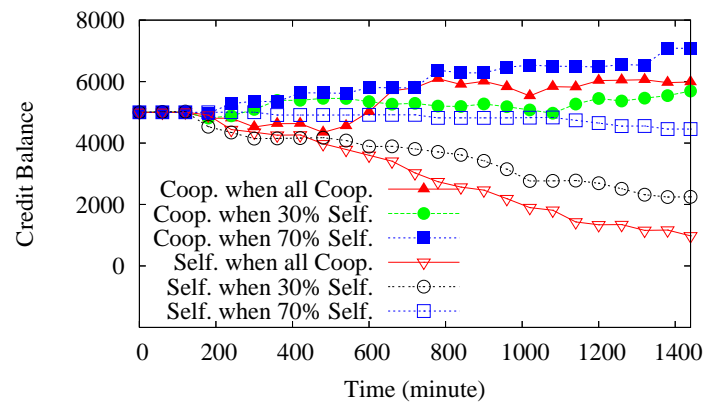

(a) No breakdown.

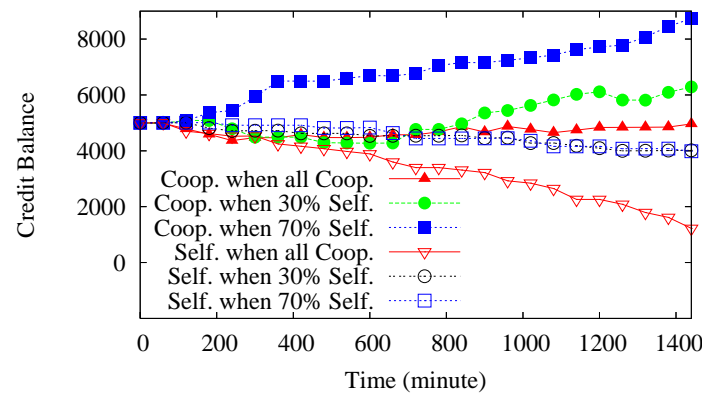

(b) Having risk of breakdown.

Fig. 4. Credit balance of node 13 in a network with 20 nodes. Six cases are compared: A) Behaving cooperatively when all the other nodes are cooperative; B) Behaving cooperatively when $30 \%$ nodes are selfish; C) Behaving cooperatively when $70 \%$ nodes are selfish; D) Behaving selfishly when all the other nodes are cooperative; E) Behaving selfishly when $30 \%$ nodes are selfish; F) Behaving selfishly when $70 \%$ nodes are selfish. The figures demonstrate that the node get higher credit balance when behaving cooperatively, and selfish behaviors have no way to increase the credit balance.

Figure 3 shows the cumulative distribution function (CDF) of the achieved cumulative utilities for 400 tracked node records. This result is composed of 10 repeated simulations with different random seeds. Each simulation is on a network with 40 nodes. The figures show the results when all nodes are cooperative and when some of them are selfish. In the latter case, we consider two situations, in which $30 \%$ and $70 \%$ of the nodes are selfish. We observe that the cumulative utilities achieved by collectively being cooperative are higher than those of partially being selfish. Intuitively, this is because when more nodes are cooperative, nodes get more opportunities to forward messages, which results in getting more utilities. These figures again demonstrates that being cooperative is better than being selfish in getting utility.

Figure 4 shows the credit balances of node 13 in a 20node network, with and without a risk of breakdown. We also note that the results for the other players are similar to that of player 13. In the figures, six different cases are compared: A) Behaving cooperatively when all the other nodes are cooperative; B) Behaving cooperatively when $30 \%$ nodes are selfish; C) Behaving cooperatively when $70 \%$ nodes are selfish; D) Behaving selfishly when all the other nodes are cooperative; E) Behaving selfishly when $30 \%$ nodes are selfish; F) Behaving selfishly when $70 \%$ nodes are selfish.
We can observe that: 1) being cooperative always achieves a higher credit balance than being selfish, when the other nodes' behaviors are given; 2) the credit balance is always decreasing with the time, if the node behaves selfishly by only sending her own message. Therefore, these results again demonstrate that being cooperative is better than being selfish.

From the results presented above, we can conclude that the best strategy of the nodes is to always behave cooperatively, when our schemes are used.

\section{Impacts on Delivery Ratio}

Our second set of evaluations are to demonstrate that our schemes improve the delivery ratio of probabilistic routing in face of selfish nodes. As we have mentioned, selfish behavior of nodes can lower the network performance. In contrast, our schemes can prevent selfish behavior of nodes, and thus can boost the network performance in terms of delivery ratio in face of selfish nodes.

Figure 5 shows the average delivery ratio as a function of the number of nodes in the network when all nodes behave cooperatively and when some of the nodes behave selfishly. In the latter case, we consider two cases, in which $30 \%$ and $70 \%$ of the nodes behave selfishly. The figures show that the average delivery ratio increases with the number of nodes in the network, and the highest delivery ratio is always 


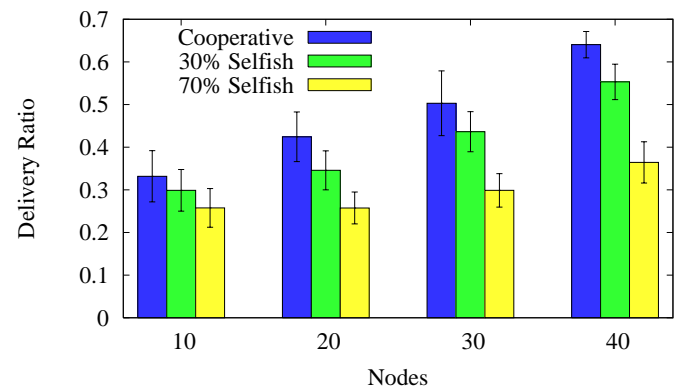

(a) No breakdown.

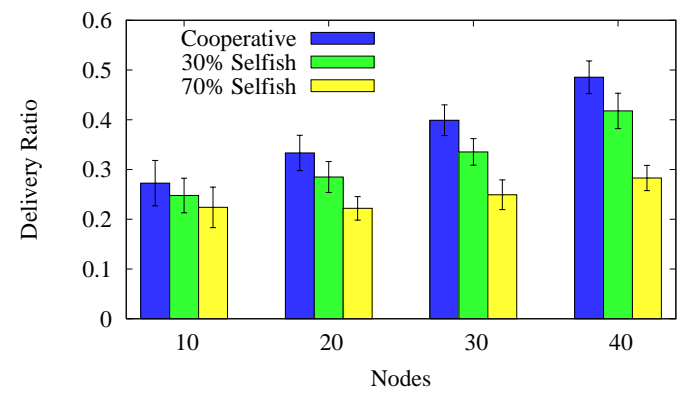

(b) Having risk of breakdown.

Fig. 5. Average delivery ratio as a function of the number of nodes in the network. Standard deviations are shown using lines. Three cases are compared: A) $100 \%$ nodes behave cooperatively; B) $30 \%$ nodes behave selfishly; C) $70 \%$ nodes behave selfishly. The figures show that the highest delivery ratio is achieved by $100 \%$ being cooperative, which can be guaranteed by our incentive schemes.

achieved by $100 \%$ being cooperative, which can be guaranteed by our incentive schemes. However, the larger number of nodes is, the more significant advantage of using our schemes is. Particularly, when the length of the game is known, our schemes achieves $11.1-22.8 \%$ and $28.8-75.8 \%$ gain in delivery ratio in the cases where $30 \%$ and $70 \%$ of the nodes behave selfishly, respectively; when there is a risk of breakdown, our schemes achieves $10.1-19.0 \%$ and $21.7-71.6 \%$ gain in delivery ratio in the cases where $30 \%$ and $70 \%$ of the nodes behave selfishly, respectively.

\section{RELATED WORK}

In this section we briefly review the related works on routing in opportunistic networks and cooperation in wireless networks.

\section{A. Routing in Opportunistic Networks}

Routing in opportunistic networks has been studied for many years. Some early works (e.g., [33], [34], [39]) use epidemic routing to distribute messages to destinations.

Later a group of works, namely probabilistic routing, extend the idea of epidemic routing by estimating the probability of each link to destination and use this information to decide whether it should store the packet and wait for a better chance as well as to decide which nodes to forward. In particular, PROPHET [27] estimates a probabilistic metric called delivery predictability, which indicates the probability of successfully delivering a message to the destination from the local node, and forward messages to the nodes who have higher delivery predictabilities to the destination. In a similar manner, MV [8] uses past frequencies of contacts to estimate the probability of delivery. Devis et al. studied the performance of a number of different strategies for deciding which messages to exchange when two nodes meet and which messages to drop when buffer is full [13]. Kun et al. proposed a shortest expected path routing (SEPR) based on link forwarding probability in the history data [36]. LeBrun et al. proposed a method using the motion vector $(\mathrm{MoVe})$ of mobile nodes to predict their future location [24]. Messages are passed to the node that is moving closer to the destination. Ghosh et al. elegantly calculated mobility profiles of individual wireless users involved in a probabilistic movement among hubs, and used the mobility profiles to guide probabilistic routing [16].

With the help of position information, some locationbased routing algorithms emerged. Greedy Perimeter Stateless Routing (GPSR) [23] uses Most Forwarding Progress within radius $R$ (MFR) to greedily forward messages. Jain et. al. use partial location information to achieve suboptimal routing decision [19]. Authors in [17], [31] proposed to use local information, which is the history of other nodes it has encountered in the past, to eliminate the cost to update location state.

Another group of works use certain knowledge about the network to direct the route of messages. For example, Jain et al. [20] defined four knowledge oracles, which represent some particular knowledge of network, and presented a routing algorithm based on the oracles. Chen and Murphy introduced utility functions to describe the usefulness of a host as the next hop for forwarding a message [12]. Burgess et al. proposed MaxProp to schedule messages transmission and determines which messages should be deleted when buffer space is full, based on the path likelihoods to nodes according to historical data, as well as acknowledgments [7].

To improve the performance of routing protocols in opportunistic networks, it is necessary to better understand the underlying user mobility. Some researchers tried to find practical mobility model to assist reproducing user movement in simulations [11], [15], [25], [37].

\section{B. Cooperation in Wireless Networks}

A considerable amount of work has been done on the cooperation problems in wireless networks. There are two major approaches: route selection based approach and packet forwarding based approach. The route selection based approaches (e.g., [3], [40]-[42], [45]) calculates the lowest cost path despite of the fact that selfish nodes can make false claims about their costs. Most works in the category only work when there are contemporaneous end-to-end routes. In contrast, the packet forwarding based approaches (e.g., [5], [9], [28], [44]) stimulate selfish nodes to forward packets. Here, we focus on the later category, since our work falls into it.

The earliest packet forwarding based approach was presented by Marti et al. [28]. Their major contribution is 
proposing a watchdog and a pathrater, which monitor the reputation of nodes. Similarly, Buchegger and Le Boudec proposed a state machine, which is updated according to the observation and received reports of other nodes' behavior [5], [6]. Srinivasan et al. proposed generous TIT-FOR-TAT and showed that this strategy leads to a Nash Equilibrium [35]. Jaramillo and Srikant used the theory of repeated game to study packet forwarding [22]. Among many other interesting results, they proved that their scheme DAWIN is optimal in their repeated game model. Recently, Shevade et al. proposed a TIT-FOR-TAT-based incentive mechanism to let nodes reward or punish their neighbors based on the history they have observed in the routing process [32]. In common, these works enforce packet forwarding by constructing reputation systems.

In contrast, some other works use credit, or virtual money, as compensation for participating the game and forwarding packets. Buttyan and Hubaux was the first to use virtual money for the packet forwarding [9]. Their solution needs the help of a piece of tamper-proof hardware on each node. Zhong et al.'s Sprite [44] is another simple credit-based solution but it does not require tamper-proof hardware. Another solution to this problem was due to Jakobsson et al., using a micropayment scheme [21]. Zhong et al. combined problems of route selection and packet forwarding and designed a protocol using an integrated approach of game theory and cryptography [43]. Lee et al. presented a secure incentive framework for commercial ad dissemination in vehicular networks [26].

\section{CONCLUSIONS AND Future WORK}

In this paper, we have presented novel and practical schemes to integrate incentive compatibility into a class of probabilistic routing protocols for opportunistic networks. We have integrated our schemes with MV routing and evaluated them using GloMoSim. Evaluation results have shown that: A) behaving cooperatively is to the best interest of each node under our schemes; B) our incentive schemes can substantially improve network delivery ratio (10.1\%-75.8\% in our evaluated settings) in the presence of selfish nodes.

Since this work focuses on unicast probabilistic routing, an interesting direction of future work is designing similar practical schemes that can work with multicast and broadcast probabilistic routing. Noting that collusion is an increasingly important problem in user contributed wireless networks, another attractive direction of future work is designing collusionresistent probabilistic routing protocol.

\section{REFERENCES}

[1] The American Heritage Dictionary of the English Language, Fourth Edition. Houghton Mifflin Company, 2006.

[2] E. Adar and B. A. Huberman, "Free riding on Gnutella," First Monday, vol. 5 , no. 10 , Oct. 2000.

[3] L. Anderegg and S. Eidenbenz, "Ad hoc-VCG: a truthful and costefficient routing protocol for mobile ad hoc networks with selfish agents," in Proceedings of the Ninth International Conference on Mobile Computing and Networking (MobiCom), San Diego, CA, Sep. 2003.

[4] N. Ben Salem, L. Buttyan, J.-P. Hubaux, and M. Jakobsson, "A charging and rewarding scheme for packet forwarding in multi-hop cellular networks," in Proceedings of the Fourth ACM Symposium on Mobile Ad Hoc Networking and Computing (MobiHoc), Annapolis, MD, Jun. 2003.
[5] S. Buchegger and J.-Y. Le Boudec, "Nodes bearing grudges: Towards routing security, fairness, and robustness in mobile ad hoc networks," in Proceedings of the Tenth Euromicro Workshop on Parallel, Distributed and Network-based Processing (EUROMICRO-PDP), Jan. 2002.

[6] — , "Performance analysis of the CONFIDANT protocol: Cooperation of nodes - fairness in dynamic ad-hoc networks," in Proceedings of the Eighth International Conference on Mobile Computing and Networking (MobiCom), Atlanta, GA, Nov. 2002.

[7] J. Burgess, B. Gallagher, D. Jensen, and B. N. Levine, "MaxProp: Routing for vehicle-based disruption-tolerant networks," in Proceedings of 25th Annual IEEE Conference on Computer Communications (INFOCOM), Barcelona, Spain, Apr. 2006.

[8] B. Burns, O. Brock, and B. N. Levine, "MV routing and capacity building in disruption tolerant networks," in Proceedings of 24th Annual IEEE Conference on Computer Communications (INFOCOM), Miami, FL, Apr. 2005.

[9] L. Buttyan and J.-P. Hubaux, "Enforcing service availability in mobile ad-hoc WANs," in Proceedings of the First ACM Workshop on Mobile Ad Hoc Networking and Computing (MobiHoc), Boston, Massachusetts, Aug. 2000.

[10] - Security and Cooperation in Wireless Networks. Cambridge University Press, 2007.

[11] T. Camp, J. Boleng, and V. Davies, "A survey of mobility models for ad hoc network research," in Wireless Communications and Mobile Computing (WCMC): Special issue on Mobile Ad Hoc Networking: Research, Trends and Applications, vol. 2, no. 5, 2002, pp. 483-502.

[12] X. Chen and A. L. Murphy, "Enabling disconnected transitive communication in mobile ad hoc networks," in Proceedings of The Sixth DIALM-POMC Joint Workshop on Foundations of Mobile Computing, Aug. 2001.

[13] J. A. Davis, A. H. Fagg, and B. N. Levine, "Wearable computers as packet transport mechanisms in highly-partitioned ad-hoc networks," in Proceedings of International Symposium on Wearable Computers, Oct. 2001.

[14] S. Eidenbenz, G. Resta, and P. Santi, "Commit: A sender-centric truthful and energy-efficient routing protocol for ad hoc networks with selfish nodes," in Proceedings of the 19th International Parallel and Distributed Processing Symposium (IPDPS), Denver, CO, Apr. 2005.

[15] J. Ghosh, M. J. Beal, H. Q. Ngo, and C. Qiao, "On profiling mobility and predicting locations of wireless users," in Proceedings of the Second International Workshop on Multi-hop Ad Hoc Networks (REALMAN), May 2006.

[16] J. Ghosh, H. Ngo, S. Yoon, and C. Qiao, "On a routing problem within probabilistic graphs," in Proceedings of 26th Annual IEEE Conference on Computer Communications (INFOCOM), Anchorage, AK, May 2007.

[17] M. Grossglauser and M. Vetterli, "Locating nodes with EASE: Last encounter routing in ad hoc networks through mobility diffusion," in Proceedings of 22nd Annual IEEE Conference on Computer Communications (INFOCOM), San Francisco, CA, Apr. 2003.

[18] C.-M. Huang, K. chan Lan, and C.-Z. Tsai, "A survey of opportunistic networks," in Proceedings of the 22nd International Conference on Advanced Information Networking and Applications - Workshops, Mar. 2008.

[19] R. Jain, A. Puri, and R. Sengupta, "Geographical routing using partial information for wireless ad hoc networks," IEEE Personal Communications, vol. 8, no. 1, pp. 48-57, Feb. 2001.

[20] S. Jain, K. Fall, and R. Patra, "Routing in a delay tolerant network," in Proceedings of ACM SIGCOMM 2004 Conference on Applications, Technologies, Architectures, and Protocols for Computer Communications, Portlan, Oregon, Aug. 2004.

[21] M. Jakobsson, J.-P. Hubaux, and L. Buttyan, "A micro-payment scheme encouraging collaboration in multi-hop cellular networks," in Proceedings of the Seventh International Financial Cryptography Conference $(F C)$, Gosier, Guadeloupe, Jan. 2003.

[22] J. Jaramillo and R. Srikant, "Darwin: Distributed and adaptive reputation mechanism for wireless ad-hoc networks," in Proceedings of The Thirteenth International Conference on Mobile Computing and Networking (MobiCom), Montreal, Canada, Sep. 2007.

[23] B. Karp and H. T. Kung, "GPSR: Greedy perimeter stateless routing for wireless networks," in Proceedings of the Sixth International Conference on Mobile Computing and Networking (MobiCom), Boston, MA, Aug. 2000 . 
[24] J. LeBrun, C.-N. Chuah, D. Ghosal, and M. Zhang, "Knowledge-based opportunistic forwarding in vehicular wireless ad hoc networks," in Proceedings of the 62nd Semiannual Vehicular Technology Conference (VTC), May 2005

[25] J.-K. Lee and J. C. Hou, "Modeling steady-state and transient behaviors of user mobility:: formulation, analysis, and application," in Proceedings of The Seventh ACM Symposium on Mobile Ad Hoc Networking and Computing (MobiHoc), Jun. 2006.

[26] S. Lee, G. Pan, J. Park, M. Gerla, and S. Lu, "Secure incentives for commercial ad dissemination in vehicular networks," in Proceedings of The Eighth ACM Symposium on Mobile Ad Hoc Networking and Computing (MobiHoc), Sep. 2007.

[27] A. Lindgren, A. Doria, and O. Schelen, "Probabilistic routing in intermittently connected networks," in Proceedings of the Fourth ACM Symposium on Mobile Ad Hoc Networking and Computing (MobiHoc), Annapolis, MD, Jun. 2003.

[28] S. Marti, T. Giuli, K. Lai, and M. Baker, "Mitigating routing misbehavior in mobile ad hoc networks," in Proceedings of the Sixth International Conference on Mobile Computing and Networking (MobiCom), Boston, MA, Aug. 2000.

[29] A. Muthoo, Bargaining Theory with Applications. The Cambridge University Press, 1999.

[30] M. J. Osborne and A. Rubenstein, A Course in Game Theory. MIT Press, 1994.

[31] N. Sarafijanovic-Djukic and M. Grossglauser, "Last encounter routing under random waypoint mobility," in Third International IFIP-TC6 Networking Conference (Networking), May 2004.

[32] U. Shevade, H. H. Song, L. Qiu, and Y. Zhang, "Incentive-aware routing in DTNs," in Proceedings of the 16th International Conference on Network Protocols (ICNP), Orlando, FL, Oct. 2008.

[33] T. Small and Z. J. Haas, "The shared wireless infostation model - a new ad hoc networking paradigm (or where there is a whale, there is a way)," in Proceedings of the Fourth ACM Symposium on Mobile Ad Hoc Networking and Computing (MobiHoc), Annapolis, MD, Jun. 2003.

[34] T. Spyropoulos, K. Psounis, and C. S. Raghavendra, "Spray and wait: An efficient routing scheme for intermittently connected mobile networks," in SIGCOMM workshop on Delay-tolerant networking (WDTN), 2005.

[35] V. Srinivasan, P. Nuggehalli, C.-F. Chiasserini, and R. Rao, "Cooperation in wireless ad hoc networks," in Proceedings of 22nd Annual IEEE
Conference on Computer Communications (INFOCOM), San Francisco, CA, Apr. 2003.

[36] K. Tan, Q. Zhang, and W. Zhu, "Shortest path routing in partially connected ad hoc networks," in Proceedings of IEEE 2003 Global Communications Conference (GLOBECOM), Nov. 2003.

[37] C. Tuduce and T. Gross, "A mobility model based on wlan traces and its validation," in Proceedings of 24th Annual IEEE Conference on Computer Communications (INFOCOM), Miami, FL, Apr. 2005.

[38] UCLA Parallel Computing Laboratory, "Glomosim: Global mobile information systems simulation library. http://pcl.cs.ucla.edu/projects/glomosim/."

[39] A. Vahdat and D. Becker, "Epidemic routing for partially-connected ad hoc networks," Duke University, Technical Report CS-200006, 2000.

[40] W. Wang, S. Eidenbez, Y. Wang, and X.-Y. Li, "Ours-optimal unicast routing systems in non-cooperative wireless networks," in Proceedings of The Twelfth International Conference on Mobile Computing and Networking (MobiCom), Los Angeles, Sep. 2006.

[41] W. Wang, X.-Y. Li, and Y. Wang, "Truthful multicast in selfish wireless networks," in Proceedings of the Tenth International Conference on Mobile Computing and Networking (MobiCom), Philadelphia, PA, Sep. 2004.

[42] F. Wu, T. Chen, S. Zhong, L. E. Li, and Y. R. Yang, "Incentivecompatible opportunistic routing for wireless networks," in Proceedings of The Fourteenth International Conference on Mobile Computing and Networking (MobiCom), San Francisco, CA, Sep. 2008.

[43] S. Zhong, L. E. Li, Y. G. Liu, and Y. R. Yang, "On designing incentive-compatible routing and forwarding protocols in wireless adhoc networks-an integrated approach using game theoretical and cryptographic techniques," in Proceedings of The Eleventh International Conference on Mobile Computing and Networking (MobiCom), Cologne, Germany, Sep. 2005.

[44] S. Zhong, J. Chen, and Y. R. Yang, "Sprite, a simple, cheat-proof, creditbased system for mobile ad-hoc networks," in Proceedings of 22nd Annual IEEE Conference on Computer Communications (INFOCOM), San Francisco, CA, Apr. 2003

[45] S. Zhong and F. Wu, "On designing collusion-resistant routing schemes for non-cooperative wireless ad hoc networks," in Proceedings of The Thirteenth International Conference on Mobile Computing and Networking (MobiCom), Montreal, Canada, Sep. 2007. 